\title{
7 Relocation and Relationships
}

\section{Encounters on the Way}

Years back, during childhood days, the radio in the car would blare strange words into the hot dusty summer afternoon. Heia Safari, a male voice crooned, and what that meant, and was, never became very clear to the child on the back seat of the car. The father, a lover of jazz and Bebop, hastily switched to another station, obviously finding Heia Safari unbearable. Sometime later, a find in the great-aunt's attic: Heia Safari. A book on Africa, written for the youth.

Years and years later, a man waited in front of the Kenyan beach resort and said: Safari ham wir schon? 'Safari done already?' The hotel's souvenir shop offered various items that bore images of the 'big five', the large mammals that are presented as the highlights of all the sightseeing and photography. A decorative plate with lion, buffalo, elephant, rhino and leopard would later testify that nature had been seen, a journey had been made, wilderness conquered.

The man continued his attempt to establish a conversation by asking a few more things about how we liked it here and why we had skipped the national park. Most tourists came on a package tour which usually included a safari in one of the parks first, with animal sightings and Maasai village visits, and then continued with a week on the beach, with an all-you-can-eat buffet and pool gymnastics. Not easy to get into contact with the tourists, he explained politely, who do not seem to trust the beach vendors and local entrepreneurs and who do everything with their tour guides. He was from the village that was no longer a village because it had been replaced by beach resorts and tourist malls. The rejections of the majority of the package tourists felt like a second expulsion from home, after the expulsion from the land itself. It created frustration and violence in the ways in which conversations at the beach were ended, indirect communications in German and English, but rarely Swahili. The things that were felt and perceived remained unsaid, as if these homeless conversations had nothing to do any longer with the feeling of a place, the 
sensual perception of the bodies that populated it. Yet the language of the senses is ubiquitous in the safari world, which remains a colonial sphere.

\section{Sensual Stranger}

'My young head was in a ferment of new things seen and realised at every turn and - that I might in any way keep abreast of the flood - it was necessary that every word should tell, carry, weigh, taste and, if need were, smell' (Kipling, 1990: 120). Rudyard Kipling today seems to be the epitome of the imperial, and the colonial. Not just his poems and novels, but his life, as told in the autobiographical text from which this fragment is taken, provide deep insights into what we could call the essence of the colonial experience. As a young man, Kipling seems overwhelmed by the plethora of words and the concepts embedded in them, and has, in the extreme, even to smell those words that he would otherwise not be able to grasp. The confusion that emerges out of diversity and the complexity of what is said and what cannot be said may have been unsettling, but it remained a significant working tool for Kipling.

Getting over this crucial confusion and turning diversity into poetry also meant that the words' smells and weight would be understood, or felt, by others who hadn't been where Kipling had been - in Cape Town, Allahabad, Simla. Writing about oneself as part of a giant commotion intellectually, culturally and geographically - is a common trope in autobiographical texts of the period and requires us to look at the entire body of the writer: ears, tongue, nose, hands, heat and the moisture on the skin, the smell of food on his plate, the ache of limbs after a day's walk. Kipling is extremely precise in his descriptions of sensual perceptions and his bodily reactions to them, and it is striking how his physical presence is there in these texts. We could say almost the same about Flaubert as he writes about his journey to Egypt, about visiting slave markets and brothels.

But what is this really about - is this a matter of genre and a colonial trope? Are the bodies of these Northern visitors to the Orientalized spaces of the colonized parts of the world embedded in a more compelling sensuality? Does the notion of smell and the intensity of feeling convey a sense of uncontrolled diversity, foreignness embedded in the foreign? Is the otherwise closed and controlled body of the European traveller turned into an atavistic body, with smell suddenly becoming a primary sense (Beer, 2000)? In principle, it seems, these texts and their motifs are just exquisite examples of colonial mimesis, '[...] a process and [...] a practice of the colonizers themselves that could bear productively on colonial relations of power' (Ferreira, 2012).

Perhaps we have to change our perspective in order to be able to grasp the ubiquity of such motifs and metaphors. What precisely constitutes these diverse spaces? Who lives in them and how do their inhabitants speak about themselves? What makes these imagined fringes of formerly colonized societies and spaces smelly, messy and confusing? Why do they 
require their visitors to think about their sensual reactions and bodies in this intense way? Having been interpreted as an expression of Orientalism, sensuality in colonial writing is very much looked at within a Western framework. But perhaps, thinking of the beach and the safari connected to each other through tourist locomotion, we can draw a line from writing in colonial settings to the plurality of speech at these sites.

\section{A Moved Village}

Villages that stood in the way of the creation of a national park, a dam or a resort abound. The village of Kiryandongo, just outside Murchison Falls National Park in Uganda, and just a few kilometres from the provincial town of Masindi, was relocated by the British in the 1950s when the national park was implemented. Kiryandongo is claimed to be a Chopi village, owned by a group of people whose ancestors originally lived close to the falls, and located in a region that is characterized by pervasive multilingualism, high linguistic diversity and a very feeble correlation between language and (ethnic) identity concepts. Some Chopi speakers told us they had always lived in 'mixed' villages, then and now, and felt at ease with other people, and therefore that they had easily adapted to the new settling area since the mid-20th century.

This, however, was a very ideologically framed way of dealing with their history, because many Chopi clearly felt that they weren't properly compensated, and because relocation was not the only time that other people had interrupted their lives. During the war of the Lord's Resistance Army against the Ugandan state, villages like Kiryandongo suffered tremendously, with many children being abducted to become soldiers. But experiences of crisis in this area date back centuries. The area was frequently affected by slave raiders and epidemics alike (Doyle, 2006; Médard \& Doyle, 2007; Reid, 2002, 2007), and these, as well as local conflict, were responsible for the mobility that characterized the social histories of the Great Lakes regions (Kopytoff, 1987). Conflict and crisis tended to produce new settlements rather than resulting in lengthy civil war, as one of the preferred strategies in conflict resolution was migration. New settlements gained power and safety just through the inclusion of other people. Such strategies have resulted in the conceptualization and practice of multilingualism as a social and political necessity, and as a survival strategy. Current globalized power games and wars have resulted in a high fluidity of migrants and the languages they speak.

Conflict and war resulted in an influx of migrants from almost everywhere. Many villagers claimed that this did not pose any problems to them, as they were welcoming people. The fields around the village were hard to cultivate, they said, with very heavy soil, and manpower was always welcome.

Being aware of the global grasp on agriculture and tourism sites, the villagers clearly noticed that the multilingual repertoires of the area were 
becoming richer and more diverse. 'Rare' and therefore prestigious languages must have been present for a long time, such as Maltese and Polish, spoken by the inhabitants of a World War II POW camp nearby. Now, however, these languages multiplied. An interesting strategy that mirrors this situation is that the villagers have taken up counting the languages spoken in Kiryandongo. There were 58 languages present in the year 2000, and 72 in 2014. The inhabitants themselves were not counted.

\section{Speaking, Smelling and Settling}

Practices such as counting languages illustrate the complex entanglements between the local, the global and the colonial. Nobody could give us any idea of how many languages were spoken in the villages before relocation took place. Language as a separate entity, a countable unit, is hard to think about in such an environment, where the use of several ways of speaking on a daily basis is the norm. Repertoires consist of a variety of ways of speaking, which are used in specific contexts.

Considering its sociolinguistic situation, Chopi is most appropriately seen as one of several ways of speaking - one out of several options speakers are able to choose from. Concepts such as MOTHER TONGUE and NATIVE LANGUAGE (in the sense of Bonfiglio, 2010) are not easily applied. As the community receives increasing numbers of short-term migrants, who live in Kiryandongo only until they can go back or join family members elsewhere, linguistic diversity turns into a multi-layered phenomenon. This is, in principle, very much in line with observations about emerging complex diversities in global tourist spaces.

The resulting relatively new diverse contexts that characterize Kiryandongo today are two-sided. While linguistic practices may not have changed in principle, language diversity has become too complex to simply be incorporated into repertoires. These ways of speaking are therefore used, on the one hand, as 'resources [...] learned in the context of specific life spans, in specific social arenas, with specific tasks, needs and objectives defined, and with specific interlocutors' (Blommaert \& Backus, 2011: 21), but they also need to be handled in a way that relates to Northern concepts; they need to be counted, separated from each other, put into order. The presence of foreign NGOs and the accessibility of educational institutions offered by them, the church and the state most certainly invite the appropriation of linguistic diversity to language concepts employed there. Even though the notion of language as a separate entity and discernible unit stands in stark contrast to the actual ways of constructing individual, complex communicative repertoires, it could be filled with meaning. It signifies, in all its coloniality, nothing but modernity, epitomized in the ability to distinguish, to write and to list. As an interpretation of the Northern concepts and practices that came with colonialism, the acclamation of language as a named, countable unit relates Chopi localism to globalized migration and ideas about speech. 
The practice of accumulating and discursively presenting linguistic diversity is an interesting concept. Deep with meaning, it relates to a strategy that aims at continuously turning the personal into the communal, the Other into the Self: repertoires are symbolically treated not as something that 'belongs' to a person, but that represents the entire village. Of course, nobody in Kiryandongo speaks 72 languages. But the entirety of the codes represented by the current inhabitants represents what this community 'owns'. While the place and its communality are based on experiences of fragility and precarity, they are symbolically constructed as powerful and enduring by turning personal repertoires into communal diversity. Counting - thereby dividing communicative repertoires and thus LANGUAGE AS A WHOLE - in Kiryandongo makes community, combines different people's ways of speaking and turns them into one huge local sound.

This strategy also works on another level, namely the semiotics of smells, interiorities and memories. Chopi speakers, even though they emphasize their village's ownership of a communal repertoire of 72 languages, expect those who 'bring in' other languages to learn Chopi, and outsiders who learn Chopi can clearly be seen as the norm. A Chopispeaking foreigner is not received with great excitement, quite in contrast to the ability to speak other languages, an attitude which emphasizes a xenophilic language ideology. And therefore, it was not surprising to the people in Kiryandongo that we, as foreigners, were able to speak Kiswahili, or had some knowledge of Chopi, Acholi, and so on. What they didn't appreciate, after enquiring about language use in Cologne, was the German monolingual culture: using just one language on a daily basis in such a setting didn't at all elicit concepts of 'modernity' and 'development' among our interlocutors.

Diversity, however, is not just positively evaluated, but serves as an emblematic identity marker. Ubiquitous motifs and tropes of folklore include family histories as foreigner-inclusion histories, framing the Other, the foreign, the diverse as someone who can and must be incorporated (married, adopted) and thus will be turned into part of the Self.

Linguistic means that help to express, metaphorically, the relevance of turning diverse individuals into part of the communal body, are expressions of smell. Chopi, like a few other Lwoo languages, is a language with a complex terminology of smells and tastes (Storch, 2011). Some examples are:

$\begin{array}{ll}\text { tík } & \text { 'rotten, spoilt' } \\ \text { kúur } & \text { 'perfume, fried food, beer, flowers, baby, smoke' } \\ \text { mît } & \text { 'ingested food, sweets, mango' } \\ \text { keer } & \text { 'ginger, chili' } \\ \text { wác } & \text { 'sour' } \\ \text { nwée } & \text { 'fuel, stench' } \\ \text { kwôk } & \text { 'germinating food' } \\ \text { tôp } & \text { 'anything gone bad' } \\ \text { chóò } & \text { 'repulsive, disgusting' }\end{array}$


As the last term illustrates, these concepts are sometimes expressed by making use of different languages, here utilizing a Swahili lexeme. Smell terms are used in discourse in community-making contexts, e.g. beerdrinking, storytelling, resting, etc. Migrants are expected to learn them, and they often do. Most of the forms are used in discourse in a very similar way to interjections (like kèléelée 'quiet!'). They are largely absent in narratives, culinary talk, proverbs and heightened language (e.g. prayer, poetry), but surface in dialogical communication. Here, they help to situate social relations:
A néedi? 'how?'
B a-tyé ma-béc. may Isit. kom-á ò-doko lím. 1SG-stay REL-good body-POSs.ISG 3SG-become sweet 'I'm fine. May I sit? I'm re-energized.'

What is expressed here, in a greeting, but also in comments and remarks during a chat, is the ubiquity of smell in social interaction. And there is no individual way of perceiving social smell, but rather a communal one. Sensuality - in principle very personal and related to the body of the Self - is here turned to the Other, and is framed in the discursive regime of the sensuality of strangeness. This is another part of a huge metaphorical machine that turns single persons, who will perceive smells and taste in individual ways, into part of a huge communal system. There are no personal ways of perceiving odours here, but there are players who are all expected to perceive more or less the same social concept.

This metaphorical complexity is, in principle, just one way of discursively framing community making on the African Frontier. To Igor Kopytoff (1987), the mobility of people, villages, stories and repertoires results out of conflict-solving strategies, such as migration as a reaction to defeat, and the attraction of adherents by those who have started new communities and settlements. Because new conflicts could always result in the break up of a community, it was important to assemble as many followers around oneself as possible. But these people would never switch to new repertoires and fully adopt new concepts of identity; as one always had to reckon with the possibility of being ousted, it was wise to remember clan histories and various ways of speaking, as they remained potentially relevant.

This long-lasting sociopolitical setting produced concepts of standards, which were, as language ideologies, very different from the hegemonic European ideas about national language, linguistic standards and the role of the individual. In spite of their fluidity, these communities required linguistic adaptation, and local standards referred to particular combinations of ways of speaking within repertoires - in Kiryandongo, therefore, Chopi is included in most repertoires, at least to an extent that helps speakers to express the social relationships they form part of. 


\section{In the Showcase}

STANDARD in Chopi, being conceptualized as and referred to as the composition of repertoires and not the ability to reproduce a particular delimited code, applies to communicative performance and practice - very much in contrast to otherwise dominant linguistic concepts that have to do with structure, the lexicon and literacy. The latter is also claimed by language planners, educationists and politicians in Uganda. Constructing a standard and equipping its imagined speech community with an equally standardized orthography is regarded by most, in principle, as tantamount to development and modernity. But in the case of Chopi, as well as elsewhere in Lwoo-speaking East Africa and into Kenya and Tanzania, a strange twist occurs. As the expansion of Lwoo is of relatively recent date (Atkinson, 1999), Southern Lwoo languages (such as Luo in Kenya, Acholi in Uganda, and so on) are relatively similar to each other (Storch, 2005, 2006). This has fuelled linguistic debates about mother tongue education and language planning, which are basically the only social and political arenas where minority languages could become visible (Namyalo \& Nakayiza, 2014). If they are all so similar, why should separate primers, courses and media programmes be launched for all these languages? Wouldn't it suffice in principle, and make more sense economically, to provide the necessary 'tools for development' for the largest and politically most important varieties, such as Acholi, Lango and Kumam?

However, such debates do not just involve economic considerations, but also, and probably much more so, the challenges that rural linguistic diversity poses to linguistics in terms of the conceptualization of LANGUAGE as a fairly open repertoire. The practices of people living in communities such as Kiryandongo are hard to describe by means of traditional linguistics, where the aim is to describe 'a language' in order to be able, for instance, to explore social networking, contact, code-switching, etc. Having received some type of mainstream linguistic training, both project participants from abroad and local authorities engaged with language are asked to produce linguistic generalizations, which can serve as the basis for further linguistic descriptions of Chopi as a mother tongue that belongs to its speakers, thus identifying them.

And this makes sense to these speakers: Uganda's language policy, with English as the official language and Swahili, Luganda and other vernaculars as national languages, is shaped by the colonial idea of separate ethnic languages, an idea which symbolically equips these languages with considerable power:

The major challenges to the realization of linguistic rights stem from differing and often conflicting ideologies and politics and the general attitudes and perception people hold towards the diversity of languages. Hence there is a need to agitate for prestige language planning. This should, among other things, ensure continuous public language campaigns 
and lobbying policy-makers to ensure that language policies, especially those that promote linguistic rights, are translated into blueprints for action other than remaining public relation statements that serve political interests. The same type of planning will also ensure that the public understands why there is a need for people to love, develop and use their languages, not only in their homes but also in public and official domains. Since prestige planning also targets negative attitudes, similar efforts should be made to empower Uganda's indigenous languages to ensure their role in the social, political and economic development of the country. (Namyalo \& Nakayiza, 2014: 14)

Successfully showcasing Chopi as a PRESTIGE LANGUAGE, however, has not happened. And there is a specific reason for this: Chopi is not simply a way of speaking as part of liquid repertoires, but a way of speaking that has largely been unmoored from its original site. Of the old Chopi villages nothing is left, apart from the name of an upmarket safari lodge which is located near one of the old settlements: Chobe Lodge, ${ }^{1}$ a site of safari-before-beach.

The safari lodge is not a heritage site and not a suitable place for memory culture. It is, however, in all the sensuality it offers (aromatic massages, blue hour cocktails, the refreshing swimming pool), a deeply colonial site that offers various amenities of Western modernity. As such, it epitomizes all that has replaced Chopi cosmopolitanism and all that Chopi cannot be: the colonial concept of the monolingual speaker of an incipiently standardized Indigenous language directly correlates with the creation of borders. These are drawn not only around national parks, but around administered districts, ethnic groups and languages. We do not find just traces of these colonial borders on the linguistic maps of Africa; rather, they continue to underpin practices that are applied by linguists, missionaries and politicians alike.

Consequently, Chopi needs to be fixed as a language on a linguistic map, a classification table, in a development plan and as an ethnic marker in order to obtain a status that would put speakers in the position to claim their own prestige language and thereby their linguistic human rights. However, the rural melting pots whose inhabitants rely on very different concepts of language in order to successfully integrate in rural labour markets and social networks do not provide such anchoring points, and neither does a safari lodge. The ethnic emblematicity correlated with Chopi is limited to just an old rake in a dusty showcase at Kampala's National Museum (Figure 7.1).

Comments on speech, things and words are sometimes mimetic interpretations of sociolinguistic Otherness. Rudyard Kipling, living between and within both the European and the colonized societies and their respective cultural settings, comments on the strange, bewildering words that pour out of these confusing spaces and interactions by almost ingesting them. These words and the sensuality they evoke and require are 


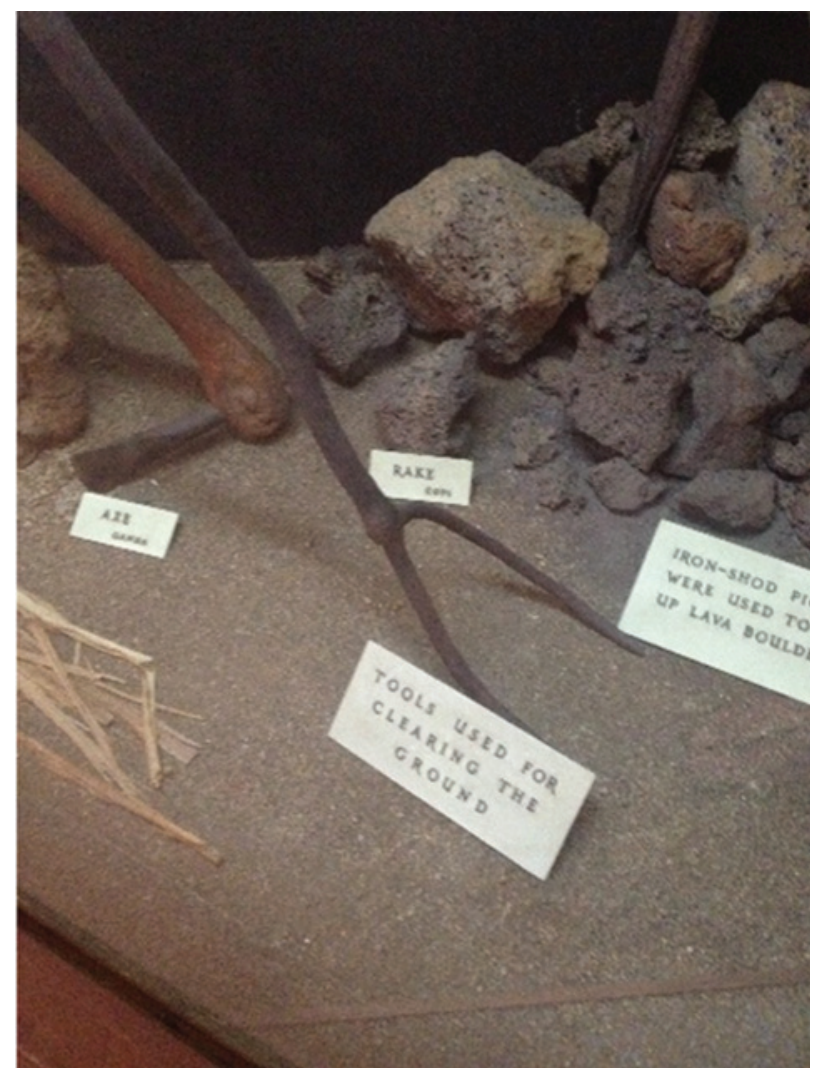

Figure 7.1 An emblematic rake, a tool for clearing the ground

framed in an imitation of the exoticism associated with the Other and their languages and narratives. By employing colonial mimesis as a narrative technique, Kipling claims possession over these Other voices which he would otherwise not be able to control.

The speakers of Chopi, and some other Luo languages, appear to do the same thing. Their strategies of counting languages as separable, discrete units in places such as Kiryandongo, and claiming the right to define standards and political status, have very little in common with the actual ways in which they use language - namely as a very open practice. By counting and defining languages they perfectly imitate the practices of the Other: the monolingual, Western, standard-speaking Other, whom they cannot and will not be in their own linguistic and social environments. These environments require a multilingual - or, better, linguistically open and mobile - speaker. But the experience of coloniality and postcolonialism provokes a strong interest in the interpretation of power represented by statistics, standards, norming and quantifying. 
In their daily interactions with those with whom they share their linguistic currencies, however, these counters and commentators handle language in another form: not as a marker of difference, but as a means of enjoying sociability, a playground of the senses and indulgence. Using a large variety of ways of speaking and combining different texts in an intricate manner is not just geared at making oneself understood in a linguistically diverse environment, but signifies openness. Strangers are not excluded by means of complex access rituals or communicative exclusivism, but are invited into communities and encouraged to indulge in verbal pleasure.

It seems as if the multilingual and multi-ethnic settlements of the African Frontier radically challenge our ideas about language and speech communities. Perhaps the inhabitants of these spaces simply use more adequate metaphors than we do. Western thinking about language is still very much based on the notion of the monolingual speaker (as a standard, a norm, a biographical construct) - a concept also reflected in the concept of MULTILINGUALISM, which somehow implies the existence of countable, separate languages stored in a person's mind. Yet words are, like desires, pleasures, the name one carries and the journeys one undertakes, part of a huge social yet transcending wealth, which is displayed in the translocated settlements around the safari destinations as well as on the beach and elsewhere, with different interests in mind and displayed for different reasons. Doesn't this encourage a slight change in our perspective, and a readiness to accept the realities of diversity that leave us as members of a tumultuous, flexible, opportunistic and vital community, and not any longer as distant observers of an endangered, or dangerous, Otherness that needs to be remade into order, that needs to undergo development?

\section{Note}

(1) The name Chobe recalls the Lwoo ethnonym chopé, as well as the Chobe Lodge in Botswana, which is one of Africa's leading hotels. 